\title{
Detecting and Characterizing Exoplanets with Direct Imaging: Past, Present, and Future
}

\author{
Beth Biller \\ Max-Planck-Institut für Astronomie, \\ Königstuhl 17, 69117 Heidelberg, Germany \\ email: biller@mpia.de
}

\begin{abstract}
The last decade has yielded the first images of exoplanets, considerably advancing our understanding of the properties of young giant planets. In this talk I will discuss current results from ongoing direct imaging efforts as well as future prospects for detection and characterization of exoplanets via high contrast imaging. Direct detection, and direct spectroscopy in particular, have great potential for advancing our understanding of extrasolar planets. In combination with other methods of planet detection, direct imaging and spectroscopy will allow us to eventually: 1) study the physical properties of exoplanets (colors, temperatures, etc.) in depth and 2) fully map out the architecture of typical planetary systems. Direct imaging has offered us the first glimpse into the atmospheric properties of young high-mass $\left(3-10 \mathrm{M}_{J u p}\right)$ exoplanets. Deep direct imaging surveys for exoplanets have also yielded the strongest constraints to date on the statistical properties of wide giant exoplanets. A number of extremely high contrast exoplanet imaging instruments have recently come online or will come online within the next year (including Project 1640, SCExAO, SPHERE, GPI, among others). I will discuss future prospects with these instruments.
\end{abstract}

Keywords. planetary systems; instrumentation: adaptive optics; techniques: high angular resolution

\section{Introduction}

In the last decade, $\sim 10$ companions and candidate companions with estimated masses $<13 \mathrm{M}_{\text {Jup }}$ have been imaged in orbit around young stars and brown dwarfs (c.f. Chauvin et al. 2005, Marois et al. 2008, Kalas et al. 2008, Lafrenière et al. 2008, Lagrange et al. 2009, Lagrange et al. 2010, Marois et al. 2010, Todorov et al. 2010, Ireland et al. 2011, Luhman et al. 2011, Kraus \& Ireland 2012, Rameau et al. 2013, Quanz et al. 2013, and Kuzuhara et al. 2013). In total, $~ 30$ companions with estimated masses $<25 \mathrm{M}_{J u p}$ have been imaged. (See http://exoplanet.eu for a compilation of these objects.) These discoveries have provided a wealth of new information about young giant planets, as well as some surprises. Prior to these detections, models predicted that young gas giant planets at young ages (10-300 Myr) would likely have cool photospheres with prominent methane absorption features (Baraffe et al. 2003, Burrows et al. 2003), i.e. that these objects would be spectral analogs to T-type brown dwarfs. However, all known directly-imaged planets at these ages (for instance, 2MASS $1207 \mathrm{~b}$ and the HR 8799 planets, Chauvin et al. 2005, Marois et al. 2008, Marois et al. 2010) have lacked methane absorption and show extremely red colors, likely due to dust clouds and/or non-equilibrium chemistry in their atmospheres (Bowler et al. 2010, Skemer et al. 2011, Barman et al. 2011a, Barman et al. 2011b, Currie et al. 2011).

Additionally, all of these companions except for $\beta$ Pic b (Lagrange et al. 2009, Lagrange et al. 2010), HR 8799e (Marois et al. 2010), and LkCa 15b (Kraus \& Ireland 2012) lie at 
B. Biller

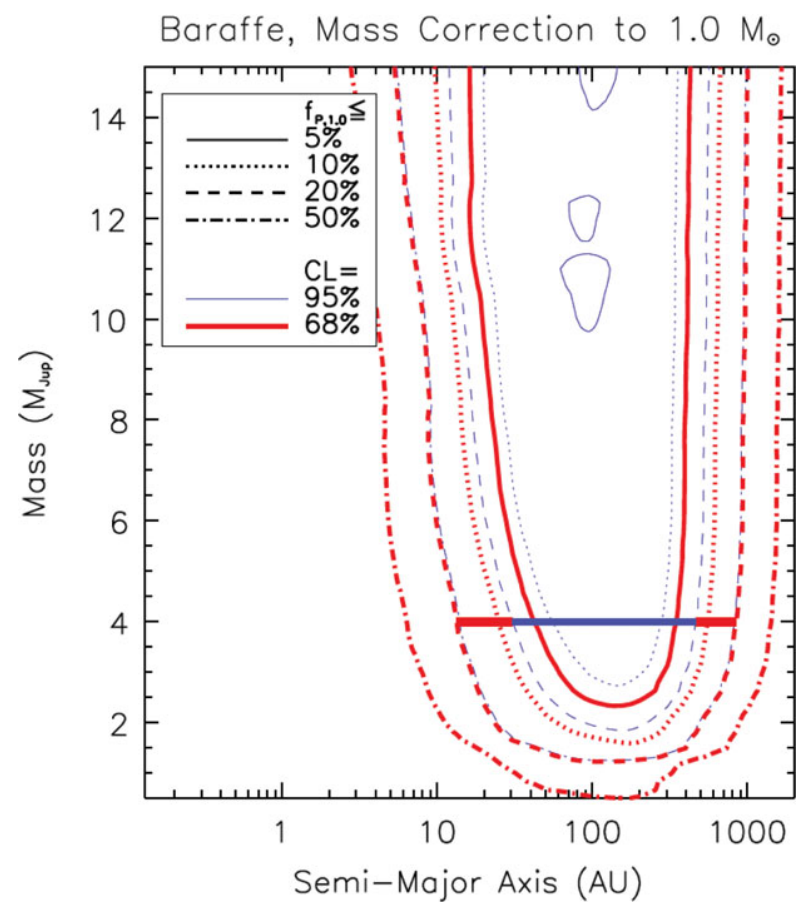

Figure 1. Figure from Nielsen \& Close 2010. Upper limit on planet fraction for $1 \mathrm{M}_{\odot}$ stars from a compilation of 118 stars observed at high contrasts, taking into account the mass scaling from Johnson et al. (2007) and using the COND models of Baraffe et al. (2003). Less than $20 \%$ of sunlike stars host $>4 \mathrm{M}_{J u p}$ planets between 30 and $466 \mathrm{AU}$ at the $95 \%$ confidence level.

projected separations greater than $20 \mathrm{AU}$, considerably wider than giant planets in our own solar system. Such widely separated companions are unlikely to be produced by insitu formation by core-accretion, which likely formed the closer-in ( $<10 \mathrm{AU})$ population of planets detected to date via radial velocity studies (Mordasini et al. 2009, Janson et al. 2012, Dodson-Robinson et al. 2009). However, given that only $\sim 10$ such companions have been imaged to date, it is premature to make statements based on such a small sample. Thus, it is a priority to discover additional companions as well as to constrain the distributions of their semimajor axes, eccentricities, masses, etc.

Further detections as well as characterization of known planets will allow us to 1) explore the physical properties of these planets (colors, temperature, and compositions) as well as 2) the architecture of their planetary systems. After discussing some of the technical aspects of direct imaging of planets and setting the stage with a quick discussion of previous and ongoing surveys, I will return to these two key themes. I will conclude with a discussion of future prospects with instruments such as GPI, SPHERE, and SCExAO.

\section{Setting the Stage - Technical Aspects and Previous Work}

\subsection{Technical Aspects of Direct Imaging}

A mature gas giant planet such as Jupiter will be $>10^{8} \times$ fainter than its host star. Young $(<100 \mathrm{Myr})$ planets are somewhat brighter, especially in the infrared, and are $10^{4}-10^{7} \times$ fainter than their host stars (e.g. Baraffe et al. 2003, Burrows et al. 2003, Fortney et al. 2008). These contrasts are in principle achievable in space or from the ground with high-order adaptive optics correction. However, even after AO correction, 


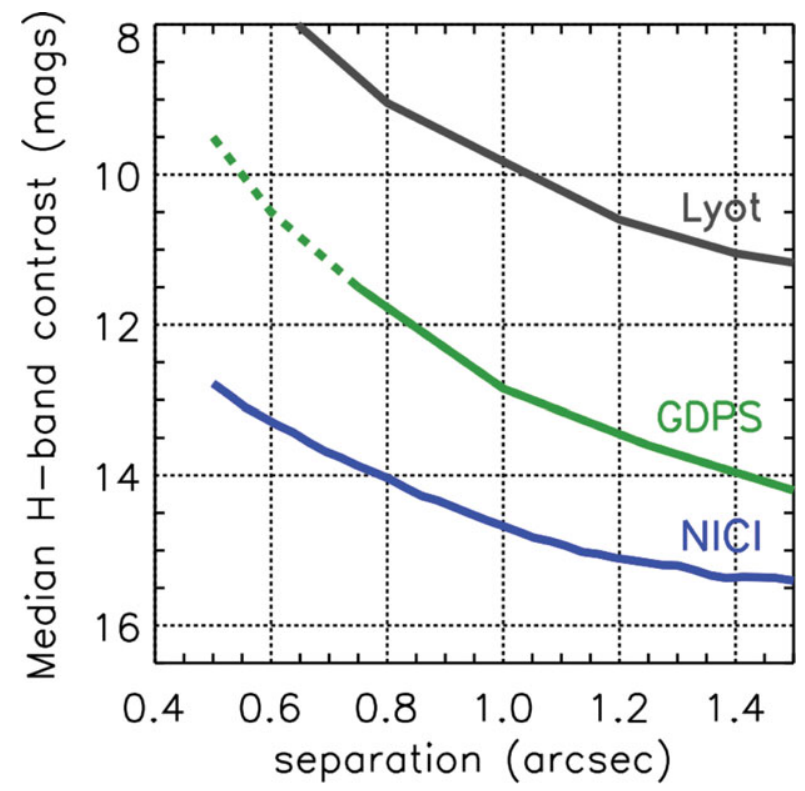

Figure 2. Figure courtesy of M. Liu and the NICI Campaign. Comparison of achieved contrast for a number of completed and ongoing surveys. Relative to the completed Lyot project (Leconte et al. 2010) and GDPS surveys (Lafrenière et al. 2007b), the NICI campaign reaches $>2$ mag deeper at separations of 0.8 ". Other ongoing coronagraphic surveys such as SEEDS, LEECH, and IDPS are reaching comparable contrasts.

speckle noise remains in high-contrast images. These "super-speckles" are generally due to imperfections in the optics themselves and appear in both ground and space data.

A number of techniques may be combined in order to attenuate starlight and suppress quasi-static speckles for direct detection of faint companions to stars. In low-strehl ratio applications, coronagraphs can be used to block out starlight. At high-strehl ratios, use of a coronagraph can correct the Airy pattern and remove a considerable amount of quasistatic pinned speckle as well, thus significantly reducing speckle noise (Sivaramakrishnan \& Oppenheimer 2006). In addition, instrumental speckle suppression techniques (e.g. azimuthal differential imaging (ADI) and spectral differential imaging (SDI)) can be used to suppress quasi-static speckles (Racine et al. 1999, Marois et al. 2006). Both SDI and ADI techniques seek to decorrelate real objects from starlight and speckles. For instance, in ADI observations the rotator is turned off at the Cassegrain focus or set to follow the elevation angle at the Nasmyth focus, allowing the field of view to rotate with parallactic angle on the sky. In a sequence of images taken at different parallactic angles, a real object will track on the sky with the parallactic angle, while speckles will move randomly. From a series of images, a reference PSF can be constructed for and subtracted from each individual image, attenuating quasi-static speckle structure. A number of different algorithms have been developed to optimize this PSF construction and subtraction, including LOCI (Locally Optimized Combination of Images) (Lafrenière et al. 2007a) as well as PCA (Primary Component Analysis) methods such as KLIP (Soummer et al. 2012), and PYNPOINT (Amara \& Quanz 2012).

\subsection{Earlier Generations of Surveys}

In the last decade, a number of deep, adaptive-optics surveys with sample sizes $>30$ stars have been completed at 8-m telescopes to search for planetary companions. Many 


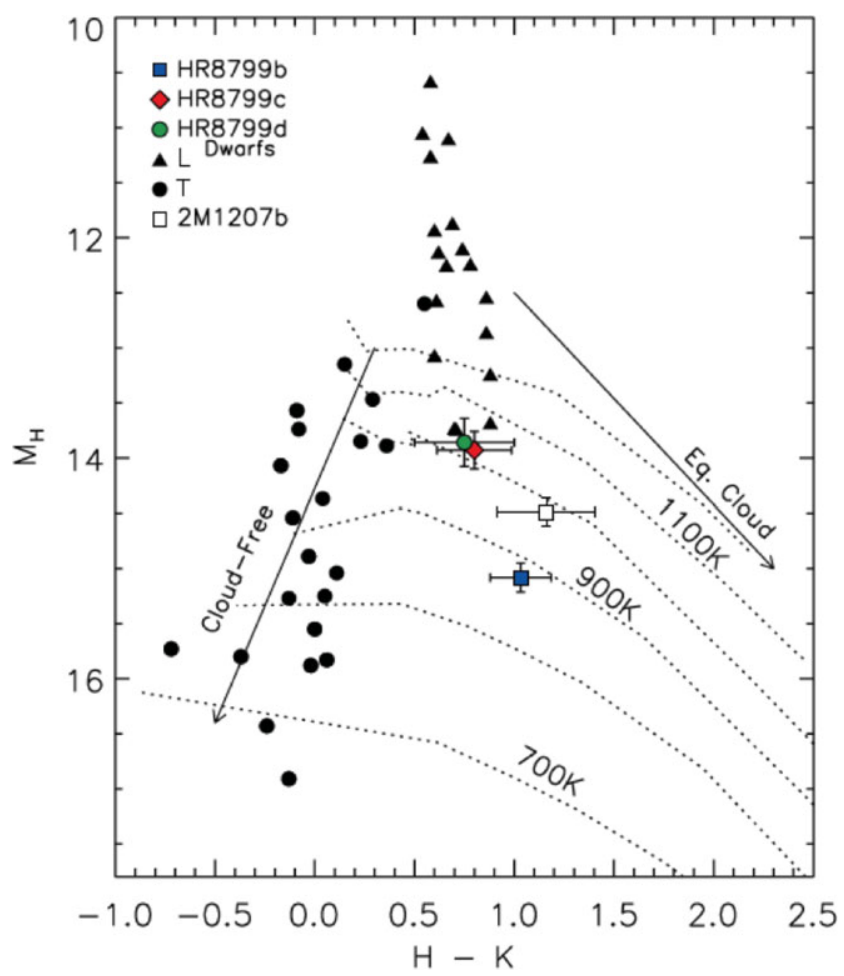

Figure 3. Figure from Barman et al. 2011b. $\mathrm{M}_{H}$ vs. $H-K$ for a number of young planets as well as comparison brown dwarfs drawn from Leggett et al. 2002 and Knapp et al. 2004. Tracks for chemical equilibrium models (radius $=1.4 \mathrm{R}_{\text {Jup }}, \log (\mathrm{g})=3.5$, mean particle size $5 \mu \mathrm{m}, \mathrm{T}_{\text {eff }}$ from $7001200 \mathrm{~K}$ ) are plotted as dotted lines. The arrows indicate the cloud-free locus (on the left side of the plot) and the location of extremely thick cloud models (on the right side of the plot). Cloud thickness increases towards the right. The extreme red colors of young planets place them closer to the L-dwarf sequence than the T-dwarf sequence due to the persistence of red dusty clouds at low temperatures and surface gravities.

of these have been conducted in the $1.6 \mu \mathrm{m} \mathrm{H}$-band or $2.2 \mu \mathrm{m} K$-band (Masciadri et al. 2005, Biller et al. 2007, Lafrenière et al. 2007b, Apai et al. 2008, Chauvin et al. 2010), while others have focused further into the infrared $(3.5-5 \mu \mathrm{m})$ in the $L, L^{\prime}$, or $M$ bands (Kasper et al. 2007, Heinze et al. 2010a, Heinze et al. 2010b, Rameau et al. 2013a.) These surveys generally median attained contrasts of $\gtrsim 10$ mag at $\gtrsim 1$ " (e.g. Biller et al. 2007) and are sensitive to $>5 \mathrm{M}_{J u p}$ planets at separations of $>20$ AU. Based on a sample of 118 stars compiled from the surveys of Masciadri et al. (2005), Biller et al. (2007), and Lafrenière et al. (2007b), Nielsen \& Close (2010) found that planets more massive than $4 \mathrm{M}_{\text {Jup }}$ are found around $<20 \%$ of FGKM stars in orbits between 22 and $507 \mathrm{AU}$, at $95 \%$ confidence (see Fig. 1). Chauvin et al. (2010) find a similar result based on a sample of 88 stars, constraining the fraction of stars with giant planets to $<10 \%$ at semi-major axes $>40$ AU for a planet distribution extended from radial velocity power laws.

\subsection{Ongoing or Recently Completed Surveys}

A number of large scale surveys (>100 stars) are ongoing or recently completed, including the NICI Campaign at Gemini-South (Liu et al. 2010, Biller et al. 2013, Wahhaj et al. 2013, Nielsen et al. 2013), the NACO Large Program using NACO at the VLT (Buenzli et al. 2010, Chauvin et al. in prep), SEEDS (Strategic Exploration of Exoplanets and Disks with Subaru) using HiCIAO at Subaru (Thalmann et al. 2009, Carson et al. 2013, 


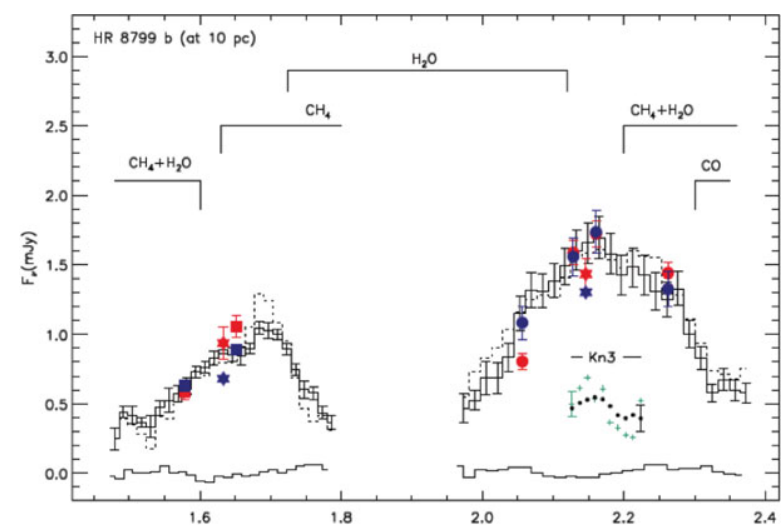

Figure 4. Figure from Barman et al. 2011a. Keck OSIRIS $H$ and $K$-band spectra of HR 8799b. A CO absorption feature is seen in $K$, but no methane absorption is apparent in either band.

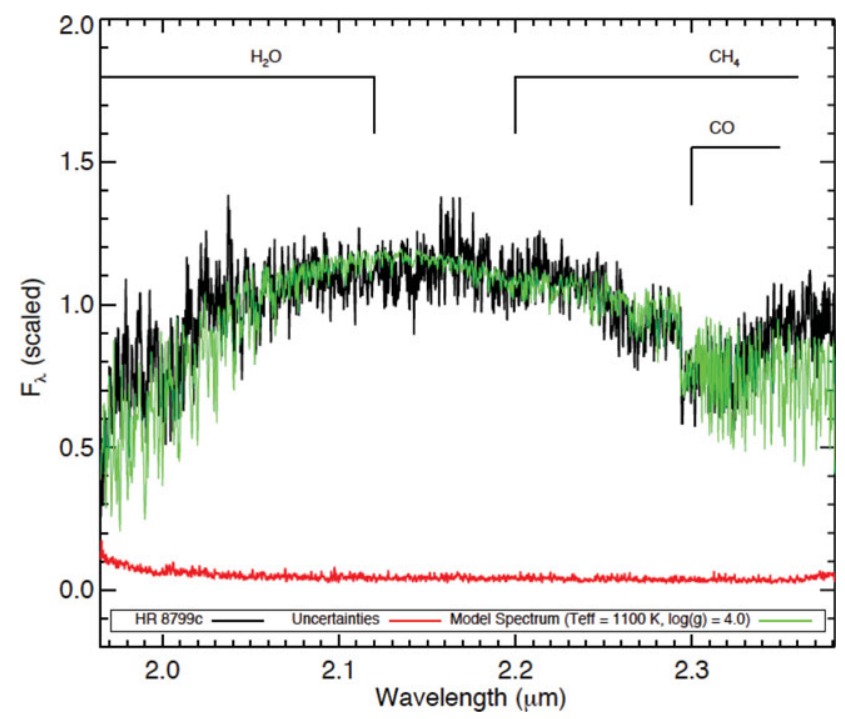

Figure 5. Figure from Konopacky et al. 2013. R = 4000 Keck OSIRIS K band spectrum of HR $8799 \mathrm{c}$, showing a clear $\mathrm{CO}$ absorption feature. From spectral fitting to this absorption feature, the $\mathrm{C} / \mathrm{O}$ ratio of HR $8799 \mathrm{c}$ appears to be supersolar, implying that this planet likely formed via core accretion.

Janson et al. 2013), LEECH at the LBT, and the International Deep Planet Survey (IDPS) using primarily Gemini and Keck (Vigan et al. 2012). These surveys combine advanced speckle suppression techniques such as ADI with coronagraphic imaging to achieve contrasts of $\Delta$ mag $>14$ at 1" (c.f. Biller et al. 2013). A comparison of current and previous surveys is presented in Fig. 2.

While the earlier generation of surveys primarily focused on solar analogues, this generation of surveys has often targeted higher mass A stars. The reasons for this are twofold: 1) a notable number of directly imaged exoplanets have been imaged around A stars (for instance, the HR 8799 planets, $\beta$ Pic, Fomalhaut) and 2) results from radial velocity surveys suggest that A stars more frequently host Jupiter mass planets than lower mass stars (Johnson et al. 2007, 2010). Another under-surveyed sample are lower mass M stars; a number of surveys are seeking to remedy this (Delorme et al. 2012, 2013, Bowler et al. 2012a, 2012b). Significant efforts are also being made to search for planets around debris 
disk stars (Wahhaj et al. 2013, Janson et al. 2013). The existence of a dust disk even after the primordial gas disk has dissipated may imply the existence of a planetary system of some form to replenish the dust. However, recent work suggests that debris disks that still retain some gas can form sharp eccentric rings solely due to dust-gas interactions and without the presence of a planet (Lyra \& Kuchner 2013).

Many ongoing surveys focus on moving group stars. Moving groups are associations of young stars (10-300 Myr) that are unconnected to regions of ongoing star-formation. These associations were not discovered until the late 1990's, as moving group members are often dispersed across a wide part of the sky (Zuckerman \& Song 2004). Moving group stars provide a unique nearby young sample with well-constrained ages and distances, vital for accurately estimating the mass, luminosity, etc. of any detected companion.

\section{Physical Properties}

The lack of methane absorption and the red IR colors of the first directly imaged planets came as a surprise (Fig. 3), as brown dwarfs with similar $\mathrm{T}_{\text {eff }}$ (thus, expected to share similar atmospheric properties and hence colors) show strong methane absorption and blue IR colors. The cause of this divergence is likely the considerable lower surface gravity of young planets compared to older, higher mass brown dwarfs. Indeed, very young, low surface gravity free-floating brown dwarfs have spectra and near-IR colors very comparable to young directly imaged planets (Faherty et al. 2013). Low surface gravity translates to very different cloud structure compared to higher surface gravity objects at the same temperatures; low surface gravity allows young planets and brown dwarfs to retain thick dust clouds in their atmospheres at temperatures at which such clouds have dissipated or sunk below the photosphere in higher surface gravity objects (Marley et al. 2012).

Direct spectroscopy of exoplanets yields even more information on the atmospheres of these objects. Spectra of HR 8799b in both the $H$ and $K$ bands show a clear lack of methane absorption as well as a CO absorption feature (Barman et al. 2011b, Bowler et al. 2010, see Fig. 4). The predominance of $\mathrm{CO}$ over $\mathrm{CH}_{4}$ strongly implies the existence of non-equilibrium chemistry. Oppenheimer et al. 2013 recently obtained simultaneous low resolution spectra of all 4 planets around HR 8799. These planets all share similar masses and formed in the same disk, yet they display intriguing variations in spectral shape, even though the broadband infrared colors for the planets are relatively similar.

The spectrum of a planet may also reveal the manner in which it formed. Konopacky et al. (2013) measure a strong CO absorption feature in their $\mathrm{R}=4000$ spectrum of HR 8799c (Fig. 5). From careful modeling of the spectrum, they derive $\mathrm{C}$ abundance, $\mathrm{O}$ abundance, and $\mathrm{C} / \mathrm{O}$ ratio for $\mathrm{HR} 8799 \mathrm{c}$. Assuming solar metallicity for the stellar host, HR 8799, and assuming the disk shares the same metallicity as the star, HR 8799c possesses a high $\mathrm{C} / \mathrm{O}$ ratio relative to solar, with $\mathrm{C}$ and $\mathrm{O}$ both comparatively depleted relative to solar. This implies strongly that the planet formed via core accretion, as a planet which formed via disk instability should share similar $\mathrm{C} / \mathrm{O}$ ratio, and $\mathrm{C}$ and $\mathrm{O}$ abundances as the natal star and disk.

Directly imaged planets also offer the opportunity to measure accurate orbits and improve mass estimates for exoplanets. Most current imaged planets are too widely separated ( $>20 \mathrm{AU}$ ) to yield good orbital determinations on reasonable $(<20$ year) timescales. However, $\beta$ Pic b, at a separation similar to Saturn in our own solar system ( 8.5 AU), holds the promise of yielding a full orbit in the relatively near future. Ongoing orbital 


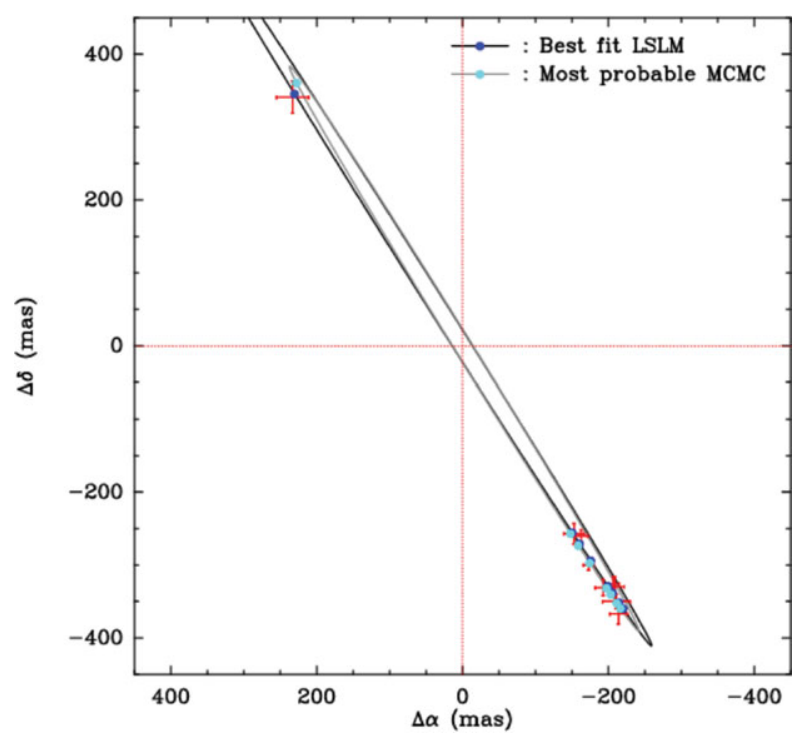

Figure 6. Figure from Chauvin et al. 2012. Best orbital fits to current $\beta$ Pic b astrometry relative to the position of $\beta$ Pic A and shown on the plane of the sky.

monitoring has already yielded estimates for a number of orbital parameters (See Fig. 6, from Chauvin et al. 2012).

\section{Architecture of Planetary Systems}

Beyond studying the physical properties of individual planets, it is important to understand the distribution of planets as a function of mass and semi-major axis in their planetary systems - i.e. system architecture. Comparatively few planets have been directly imaged considering the large sample sizes and excellent achieved contrasts $(\Delta$ mag $\sim 14$ at 1", Biller et al. 2013) of current direct imaging surveys. However, even this lack of detections provides us key information about planetary system architecture. Previous surveys demonstrated that wide giant planets $\left(>4 \mathrm{M}_{J u p},>30 \mathrm{AU}\right.$ from the host star) are rare (e.g. Nielsen \& Close 2010). Current surveys deepen and extend this result to smaller separations and lower masses. Statistical analyses have recently been published for a number of large surveys (Vigan et al. 2012, Wahhaj et al. 2013, Nielsen et al. 2013, Biller et al. 2013). As one example, I present the results from Biller et al. (2013).

Biller et al. (2013) present a subset of 80 young, moving group stars which are part of the larger ( 230 star) NICI Campaign (Liu et al. 2010). No new planetary companions were found in this sample. To statistically interpret these results, Biller et al. (2013) adapt the method established in Allen (2007) for studying stellar binarity in the context of a direct imaging survey of exoplanets, extending the power law distributions in mass and semi-major axis (with indices $\alpha$ and $\beta$ respectively) derived for RV planets by Cumming et al. (2008) to the wider separation regime ( $>10 \mathrm{AU})$ probed by direct imaging. The power law distribution in semi-major axis is only extended out to an outer cutoff, since a large reservoir of planets at very wide separations does not exist (Nielsen \& Close). The model then has four parameters: power law indices $\alpha$ and $\beta$, semi-major axis cutoff $a_{\max }$, and average number of planets per star, $F$ (used to calculate the normalization).

Using Bayesian inference techniques, constraints can be placed on these parameters based on the data from the 73 stars from Biller et al. (2013) with completed followup 
B. Biller
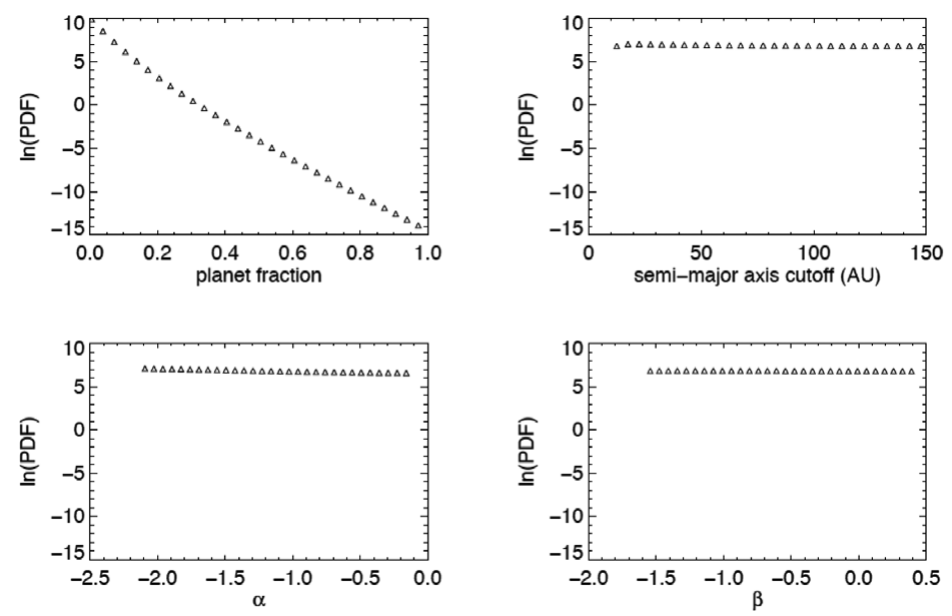

Figure 7. Figure from Biller et al. 2013. 1-d marginalized posterior pdfs including 4 free parameters and using the COND models of Baraffe et al. (2003). Posterior probability is plotted in logarithmic units. Other than for the planet frequency $\mathrm{F}$, these marginalized posteriors remain unconstrained (i.e. no clear peak or trailing off to 0 ). Thus, we have only put confidence intervals on the planet fraction for semi-major axes between 10-150 AU. At a 95.4\% confidence level, planet fraction must be $<6 \%$, marginalized over the ranges $\alpha=[-2.1,0.2], \beta=[-1.5,0.4]$, and cutoff $=[10 \mathrm{AU}, 150 \mathrm{AU}]$. Repeating the analysis with the DUSTY models of Baraffe et al. (2002) and marginalizing over the same parameter ranges, planet fraction must be $<18 \%$ at a $95.4 \%$ confidence level.

of all candidate companions and no a priori known planetary companions. Results are presented in Fig. 7. Using the COND models of Baraffe et al. (2003), $F$ must be $<6 \%$, marginalized over the ranges $\alpha=[-2.1,0.2], \beta=[-1.5,0.4]$, and cutoff $=[10 \mathrm{AU}, 150 \mathrm{AU}]$ at a $95.4 \%$ confidence level. Repeating the analysis with the DUSTY models of Baraffe et al. (2002) and marginalizing over the same parameter ranges, $F$ must be $<18 \%$ at a 95.4\% confidence level. No strong constraints can be placed on the other 3 parameters.

\section{Future Prospects}

The current generation of surveys place strong constraints on $\gtrsim 1 \mathrm{M}_{J u p}$ planets at separations $>10$ AU. The next generation of instruments will push down to smaller separations and masses. While only a handful of planets have been directly imaged to date, new instruments with much tighter inner working angles will likely yield significant numbers of discoveries. A number of $\geqslant 1 \mathrm{M}_{J u p}$ planets have been indirectly directed via the radial velocity method at semi-major axes of 5-10 AU (see compilation at exoplanet.eu). While these radial velocity detected planets are too old and faint to be directly imaged, their young counterparts should be accessible with these instruments.

A number of advanced planet-finder instruments will be on the sky within the next year. GPI at Gemini-South (Macintosh et al. 2012) will ship to Chile in summer 2013, with SPHERE at VLT (Beuzit et al. 2008) following in the autumn. SCExAO is currently undergoing commissioning at Subaru (Guyon et al. 2011). Project 1640 at the Palomar 5-m telescope has already published its first science result (Oppenheimer et al. 2013). These instruments combine extreme coronagraphy with integral field spectrographs, to enable unprecedented contrasts and novel characterization of exoplanets.

In the longer term, the extremely large telescopes (GMT, TMT, and the E-ELT) and JWST will revolutionize direct imaging and characterization of exoplanets. The 
ELTs may in fact yield the first direct image of a terrestrial sized planet. Combining results from Kepler on the frequency of short-period planets around main-sequence stars with contrast predictions for an extreme AO coronagraph on an ELT, Crossfield (2013) predicts that $\sim 10$ planets with radii of 1-8 Earth radius and equilibrium temperatures $\lesssim 400 \mathrm{~K}$ will be detectable around stars $<8$ pc from the Sun.

\section{Conclusions}

The previous and current generation of direct imaging surveys have yielded a number of interesting results regarding both the properties of extrasolar giant planets and the architectures of the planetary systems which they inhabit. Young exoplanets imaged to date have red colors indicative of clouds and/or non-equilibrium chemistry. These red colors are likely a result of the low-surface gravities of these objects, which allow red, dusty clouds to persist even at low temperatures relative to their older, high-gravity brown dwarf counterparts. Statistical analyses of large-scale planet imaging surveys have demonstrated that hot-start gas-giant $\left(>4 \mathrm{M}_{J u p}\right)$ planets are rare at $>10 \mathrm{AU}$.

\section{References}

Allen, P. R. 2007, ApJ, 668, 492

Amara, A., \& Quanz, S. P. 2012, MNRAS, 427, 948

Apai, D., Janson, M., Moro-Martín, A., et al. 2008, ApJ, 672, 1196

Baraffe, I., Chabrier, G., Allard, F., \& Hauschildt, P. H. 2002, A\& $A, 382,563$

Baraffe, I., Chabrier, G., Barman, T. S., Allard, F., \& Hauschildt, P. H. 2003, A\& A, 402, 701

Barman, T. S., Macintosh, B., Konopacky, Q. M., \& Marois, C. 2011, ApJ, 733, 65

Barman, T. S., Macintosh, B., Konopacky, Q. M., \& Marois, C. 2011, ApJ (Letters), 735, L39

Beuzit, J.-L., Feldt, M., Dohlen, K., et al. 2008, proc. SPIE, 7014

Biller, B. A., Close, L. M., Masciadri, E., et al. 2007, ApJS, 173, 143

Biller, B. A., Liu, M. C., Wahhaj, Z., et al. 2013, ApJ, 777, 160

Bowler, B. P., Liu, M. C., Dupuy, T. J., \& Cushing, M. C. 2010, ApJ, 723, 850

Bowler, B. P., Liu, M. C., Shkolnik, E. L., et al. 2012, ApJ, 753, 142

Bowler, B. P., Liu, M. C., Shkolnik, E. L., \& Tamura, M. 2012, ApJ, 756, 69

Buenzli, E., Thalmann, C., Vigan, A., et al. 2010, A\&A, 524, L1

Burrows, A., Sudarsky, D., \& Lunine, J. I. 2003, ApJ, 596, 587

Carson, J., Thalmann, C., Janson, M., et al. 2012, arXiv:1211.3744

Chauvin, G., Lagrange, A.-M., Dumas, C., et al. 2005, A $\xi A$, 438, L25

Chauvin, G., Lagrange, A.-M., Bonavita, M., et al. 2010, A\&A, 509, A52

Chauvin, G., Lagrange, A.-M., Beust, H., et al. 2012, A\&AA, 542, A41

Crossfield, I. J. M. 2013, A\&\&A, 551, A99

Currie, T., Thalmann, C., Matsumura, S., et al. 2011, ApJ (Letters), 736, L33

Cumming, A., Butler, R. P., Marcy, G. W., et al. 2008, PASP, 120, 531

Delorme, P., Gagné, J., Malo, L., et al. 2012, A\&A, 548, A26

Delorme, P., Gagné, J., Girard, J. H., et al. 2013, A\&A, 553, L5

Dodson-Robinson, S. E., Veras, D., Ford, E. B., \& Beichman, C. A. 2009, ApJ, 707, 79

Faherty, J. K., Rice, E. L., Cruz, K. L., Mamajek, E. E., \& Núñez, A. 2013, AJ, 145, 2

Fortney, J. J., Marley, M. S., Saumon, D., \& Lodders, K. 2008, ApJ, 683, 1104

Guyon, O., Martinache, F., Clergeon, C., et al. 2011, proc. SPIE, 8149,

Heinze, A. N., Hinz, P. M., Sivanandam, S., et al. 2010, ApJ, 714, 1551

Heinze, A. N., Hinz, P. M., Kenworthy, M., et al. 2010, ApJ, 714, 1570

Ireland, M. J., Kraus, A., Martinache, F., Law, N., \& Hillenbrand, L. A. 2011, ApJ, 726, 113

Janson, M., Bonavita, M., Klahr, H., et al. 2011, ApJ, 736, 89

Janson, M., Bonavita, M., Klahr, H., \& Lafrenière, D. 2012, ApJ, 745, 4 
Janson, M., Brandt, T. D., Moro-Martin, A., et al. 2013, arXiv:1306.0581

Johnson, J. A., Butler, R. P., Marcy, G. W., et al. 2007, ApJ, 670, 833

Johnson, J. A., Aller, K. M., Howard, A. W., \& Crepp, J. R. 2010, PASP, 122, 905

Kalas, P., Graham, J. R., Chiang, E., et al. 2008, Science, 322, 1345

Kasper, M., Apai, D., Janson, M., \& Brandner, W. 2007, A\&SA, 472, 321

Knapp, G. R., Leggett, S. K., Fan, X., et al. 2004, AJ, 127, 3553

Konopacky, Q. M., Barman, T. S., Macintosh, B. A., \& Marois, C. 2013, Science, 339, 1398

Kraus, A. L., Ireland, M. J., Martinache, F., \& Hillenbrand, L. A. 2011, ApJ, 731, 8

Kraus, A. L., \& Ireland, M. J. 2012, ApJ, 745, 5

Kuzuhara, M., Tamura, M., Kudo, T., et al. 2013, ApJ, accepted, arXiv:1307.2886

Lafrenière, D., Marois, C., Doyon, R., Nadeau, D., \& Artigau, É. 2007, ApJ, 660, 770

Lafrenière, D., Doyon, R., Marois, C., et al. 2007, ApJ, 670, 1367

Lafrenière, D., Jayawardhana, R., \& van Kerkwijk, M. H. 2008, ApJl, 689, L153

Lagrange, A.-M., Gratadour, D., Chauvin, G., et al. 2009, A\&A, 493, L21

Lagrange, A.-M., Bonnefoy, M., Chauvin, G., et al. 2010, Science, 329, 57

Leconte, J., Soummer, R., Hinkley, S., et al. 2010, ApJ, 716, 1551

Leggett, S. K., Golimowski, D. A., Fan, X., et al. 2002, ApJ, 564, 452

Liu, M. C., Wahhaj, Z., Biller, B. A., et al. 2010, Proc. SPIE, 7736,

Lyra, W., \& Kuchner, M. 2013, Nature, 499, 184

Luhman, K. L., Burgasser, A. J., \& Bochanski, J. J. 2011, ApJl, 730, L9

Macintosh, B. A., Anthony, A., Atwood, J., et al. 2012, Proc. SPIE, 8446 ,

Marley, M. S., Saumon, D., Cushing, M., et al. 2012, ApJ, 754, 135

Marois, C., Doyon, R., Nadeau, D., et al. 2005, PASP, 117, 745

Marois, C., Lafrenière, D., Doyon, R., Macintosh, B., \& Nadeau, D. 2006, ApJ, 641, 556

Marois, C., Macintosh, B., Barman, T., et al. 2008, Science, 322, 1348

Marois, C., Zuckerman, B., Konopacky, Q. M., et al. 2010, Nature, 468, 1080

Masciadri, E., Mundt, R., Henning, T., et al. 2005, ApJ, 625, 1004

Mordasini, C., Alibert, Y., \& Benz, W. 2009, A\&A, 501, 1139

Nielsen, E. L., \& Close, L. M. 2010, ApJ, 717, 878

Nielsen, E. L., Liu, M. C., Wahhaj, Z., et al. 2013, ApJ, 776, 4

Oppenheimer, B. R., Baranec, C., Beichman, C., et al. 2013, ApJ, 768, 24

Quanz, S. P., Amara, A., Meyer, M. R., et al. 2013, ApJ(Letters), 766, L1

Racine, R., Walker, G. A. H., Nadeau, D., Doyon, R., \& Marois, C. 1999, PASP, 111, 587

Rameau, J., Chauvin, G., Lagrange, A.-M., et al. 2013, A\&A, 553, A60

Rameau, J., Chauvin, G., Lagrange, A.-M., et al. 2013, arXiv:1305.7428

Sivaramakrishnan, A., \& Oppenheimer, B. R. 2006, ApJ, 647, 620

Skemer, A. J., Close, L. M., Szücs, L., et al. 2011, ApJ, 732, 107

Soummer, R., Pueyo, L., \& Larkin, J. 2012, ApJ (Letters), 755, L28

Thalmann, C., Carson, J., Janson, M., et al. 2009, ApJl, 707, L123

Todorov, K., Luhman, K. L., \& McLeod, K. K. 2010, ApJl, 714, L84

Vigan, A., Patience, J., Marois, C., et al. 2012, A\& $A$, 544, A9

Wahhaj, Z., Liu, M. C., Nielsen, E. L., et al. 2013, ApJ, 773, 179

\section{Discussion}

MARDLING: You didn't seem to take into account the mass of the star with your limit of $10 \mathrm{AU}$ for core accretion models.

BILlER: The limit of 10 AU I gave is specifically for solar mass stars. Higher mass stars with more massive disks may be able to form planets via core accretion at separations greater than $10 \mathrm{AU}$.

CURRIE: You mentioned the important tools needed to image planets but didn't mention powerful image processing techniques like those Christian Marois helped develop, like 
LOCI, that increase contrast. And the new techniques building on this like the SOSIE pipeline or A-LOCI can give giant gains in contrast. So within the NICI Campaign survey, how did your results leverage on exactly how you do image processing?

BILLER: Image processing techniques are certainly vital for getting the most out of ADI data. However, one thing that we found with the NICI Campaign is that different image processing techniques yielded pretty similar results on our combined ADI + SDI data. This may be because the SDI reduction is already removing most of the speckles that, say, LOCI processing of the ADI data would usually remove.

TIAN: Detection of CO in Earth-like planet's atmosphere, especially in the habitable zone: if one can detect $\mathrm{O}_{2}$ and $\mathrm{O}_{3}$ in such a planet's atmosphere, is CO detectable? Can we decide its concentration?

BILLER: If one can detect $\mathrm{O}_{2}$ and $\mathrm{O}_{3}$ in an exo-Earth atmosphere, $\mathrm{CO}$ is likely detectable too. However, such detections are quite far off. While ELTs might be able to directly image a very nearby exo-Earth, spectroscopy of such an object is certainly more than a decade away.

HomeIER: How may the claimed match to $\mathrm{T}$ dwarf templates for a planet like $8799 \mathrm{c}$ be reconciled with their still very red (L dwarf-like) SED?

BILleR: Oppenheimer et al. (2013) find L5 spectral types for HR 8799b and d. They claim a spectral type of T2 for HR 8799 c based on their $J$ band fit, however, there is clearly no methane absorption in the $H$ band for this object and the object does not look very "T"-like at all in this band. As methane absorption is what gives $\mathrm{T}$ dwarfs their characteristic blue IR colors, the absence of methane absorption in HR 8799c explains this object's red "L"-like colors. The spectrum for the closest-in planet, HR 8799e, is quite noisy, so I'd imagine any spectral type assigned to it would be quite preliminary.

KEnwORTHY: How many stars would you need to look at with an ELT M-dwarf survey to detect 3-5 Earths?

BILler: Crossfield (2013) predicts the detection of up to 10 1-8 Earth radius planets using an ELT among the $\sim 120$ main sequence stars with spectral type earlier than M7 and within 8 pc from the Earth, of which 92 are M stars. Of course, many of these detected planets will be "super-Earths" rather than truly Earth mass planets. In terms of actual Earth analogues, Crossfield (2013) predicts a $40 \%$ chance of detecting such a planet in the same 8 pc sample. 